\title{
Ask the expert
}

\section{Acute exacerbations of idiopathic pulmonary fibrosis and the role of corticosteroids}

An acute exacerbation of idiopathic pulmonary fibrosis (AEIPF) is a potentially fatal complication of an already debilitating disease. Management is currently centred on delivering excellent supportive care and identifying reversible triggers. Despite growing international awareness and collaboration, no effective therapies have been identified. Corticosteroids are often the mainstay of treatment; however, the evidence base for their use is poor. Here, we review our current understanding of the disease process and how to manage it, with a focus on the role of corticosteroid therapy.

Idiopathic pulmonary fibrosis (IPF) is characterised by progressive accumulation of abnormal extracellular matrix and an inexorable decline in lung function that is ultimately fatal [1]. As our understanding of the pathophysiology has crystallised into one predominantly of aberrant wound healing rather than chronic inflammation, the management of IPF has undergone a paradigm shift away from immune suppression towards treatments that target the maladaptive fibrotic cascade [2]. Two widely approved antifibrotic treatments, pirfenidone and nintedanib, impede the loss of vital capacity and are likely to improve survival [3-5].

Despite these advances, however, IPF remains a deadly and unpredictable disease with a guarded prognosis. Acute exacerbations of idiopathic pulmonary fibrosis (AEIPF) are a significant complication in the natural course of the disease, and herald a challenging and often life-limiting period for the patient. Aside from identifying reversible causes, treatment options are extremely limited and focus on supportive management [6]. The role of corticosteroids in AEIPF remains a significant source of debate amongst clinicians, and while they are widely used, the evidence base is poor.

\section{AEIPF definition and incidence}

An AEIPF is typified by a rapid decline in symptoms (usually $<1$ month) with new bilateral ground-glass changes on computed tomography (CT) of the chest that are not entirely explained by congestive cardiac failure or an extraparenchymal cause such as pulmonary embolus [7]. The term encompasses a broad spectrum of disease patterns, aetiologies and trajectories [8]. AEIPF was classically ascribed only to idiopathic events; yet, with the desire for a more practical definition that may facilitate clinical trial development, the term has evolved to incorporate exacerbations where a trigger is identified (figure 1) [6]. In both idiopathic and trigger-identified AEIPF, common histopathological pathways exist, typically characterised by diffuse alveolar damage or (less commonly) organising pneumonia superimposed on a background of usual interstitial pneumonia (UIP) [9].

The incidence of AEIPF varies between studies based on methodology. An event rate of four per 100 patient-years has been observed in the relatively healthy IPF populations deemed eligible for participation in placebo-controlled clinical

@ERSpublications

Acute exacerbations of IPF cause a rapid deterioration in respiratory status and are challenging to manage. With few other options, corticosteroids are widely used as standard of care; however. the evidence to support this is poor. https://bit.ly/3gvSokN
Cite as: Brereton $\mathrm{CJ}$, Jo HE Acute exacerbations of idiopathic pulmonary fibrosis and the role of corticosteroids. Breathe 2020; 16: 200086.

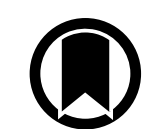

CrossMark 


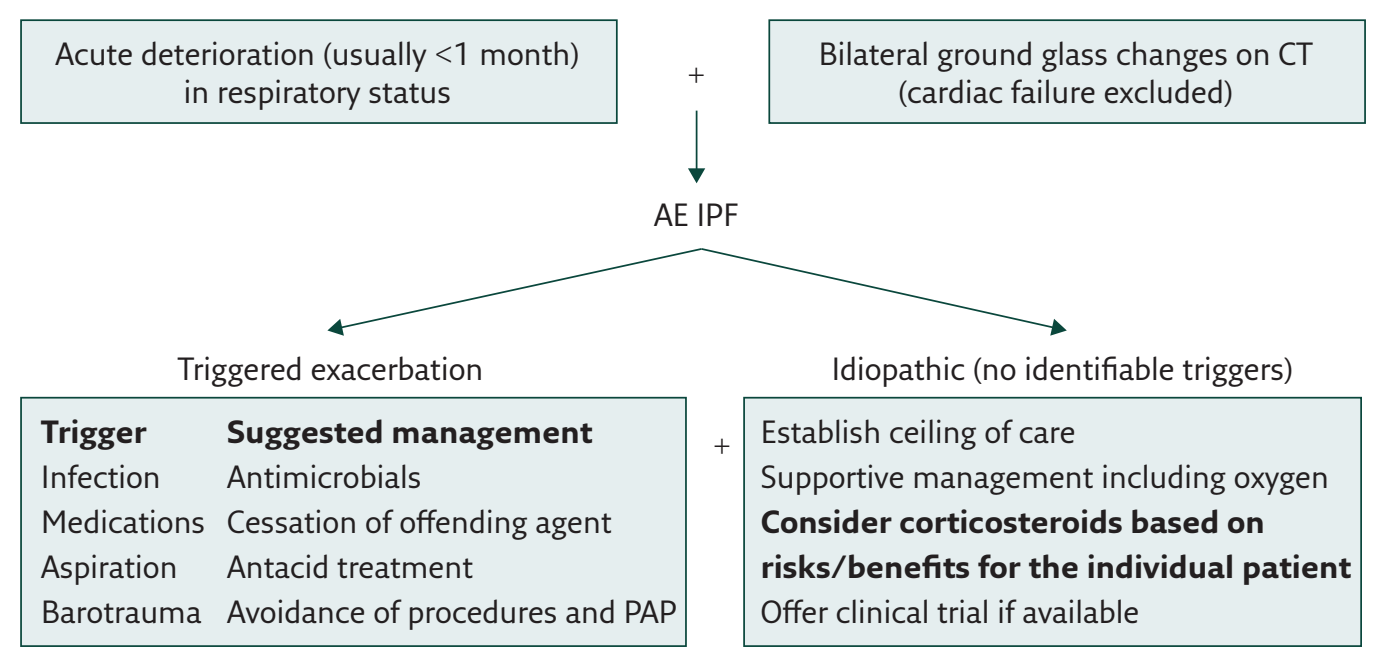

Figure 1 Suggested management of AEIPF. PAP: positive airway pressure.

trials [10]. By contrast, a 1-year incidence of 14.2\% was observed in a large retrospective cohort study, possibly reflecting a less healthy population who are more likely to be reviewed in hospital [11]. However, what remains consistent across most studies is that AEIPF is a highly fatal process. On average, half of patients hospitalised with AEIPF will not survive the event and the survivors have significantly shorter lives (15.5 months) than patients who do not experience AEIPF (60.6 months) [11].

\section{Aetiology and risk factors for AEIPF}

IPF is thought to arise from recurrent microinjuries to a dysfunctional alveolar epithelium, precipitating an aberrant fibrogenic response in the lung parenchyma of a genetically vulnerable individual [2]. In AEIPF, this epithelial injury is enhanced, either through acceleration of the recurrent microinjuries or, alternatively, a superimposed trigger [12]. In either situation, an inflammatory response develops, with an influx of immune cells and inflammatory cytokines, as seen on bronchoalveolar lavage (BAL) [13]. A variety of triggers have been implicated in this process including aspiration [14], occult infection [15], air pollution [16], thoracic surgery [17] and BAL [18]. Notably, the combination of prednisone and azathioprine has been associated with an increased risk of AEIPF, possibly secondary to increased vulnerability to infections or direct drug toxicity [19].

Aside from exposure to these underlying triggers, other risk factors associated with AEIPF likely reflect more severe disease, including a reduced or deteriorating forced vital capacity (FVC), requirement of supplemental oxygen and greater extent of UIP on CT chest [20, 21]. Furthermore, the pattern on CT of the chest confers prognostic information, with diffuse ground-glass changes associated with greater mortality than peripheral or multifocal disease [21, 22].

\section{Acute respiratory distress syndrome and the role of corticosteroids}

Similarities in clinical presentation, underlying aetiologies and histopathological appearances have led to parallels being drawn between AEIPF and acute respiratory distress syndrome (ARDS). Reflecting this, revised diagnostic criteria for AEIPF drew inspiration from the Berlin consensus statement on ARDS [23]

The impact of corticosteroids in ARDS appears favourable, with a systematic review of six large clinical trials demonstrating efficacy for their use in early moderate-to-severe ARDS [24]. In particular, the investigators observed a consistent reduction in inflammatory markers and duration of mechanical ventilation in patients treated with glucocorticoids as well as a probable reduction in mortality.

Yet, these findings cannot be extrapolated to an AEIPF cohort without a number of important caveats. In particular, the underlying UIP process weighs heavily on the IPF patient, not only as a marker of the dysregulated wound healing response but also as a pre-existing respiratory physiological limitation that is vulnerable to the adverse immune effects of corticosteroid therapy (such as superimposed infection).

\section{Corticosteroids in IPF}

Once the cornerstone of treatment, the widespread use of corticosteroids for the management of IPF has significantly diminished in the past decade. Since 2011, international consensus guidelines have strongly recommended against the use of corticosteroids, either alone or in combination with other immune-modulating therapies, for the maintenance treatment of IPF [1].

A paucity of positive evidence for their benefit was compounded by the adverse findings of the 
prematurely terminated PANTHER study, whereby their use in conjunction with azathioprine and $\mathrm{N}$-acetylcysteine resulted in increased adverse outcomes and deaths compared to placebo [19]. By 15 weeks of treatment, $32 \%$ of the combinationtherapy arm were either hospitalised or deceased, compared to $3 \%$ in the placebo arm (hazard ratio 12.11$)$. Acute exacerbations were also more common in the treatment group, occurring in five patients $(6 \%)$ compared to none in the placebo group.

Other studies have also reported indirect evidence that maintenance corticosteroid treatment may increase the likelihood of AEIPF [25]. In a metaanalysis that included six studies that reported AEIPF, the authors observed that the only study that did not permit immunosuppression had the lowest rates of acute exacerbations [10].

\section{Corticosteroids in the treatment of AEIPF}

While there has been a significant shift away from corticosteroids in the treatment of IPF, the international consensus still offers a weak recommendation for the use of corticosteroids in AEIPF. Their efficacy, however, is far from accepted, and significant debate exists regarding the appropriate type, dose and duration (if any) of corticosteroid treatment [1].

Early case reports of AEIPF where corticosteroid therapy alone was used describe a range of survival outcomes (from 4\% to 100\%), most likely reflecting the heterogeneity in patient populations and disease severity [17, 22, 26-28]. More recently, a randomised control study investigating the efficacy of thrombomodulin alfa in AEIPF treated 77 patients as baseline standard of care with pulse corticosteroid therapy followed by a taper [29]. While no significant difference was observed between the treatment $(n=40)$ and placebo $(n=37)$ groups, the overall survival at day $90(n=62,81 \%)$ was much higher than previous studies, possibly due to the clinical trial setting with a greater proportion having less severe disease. The combination of corticosteroids with a cytotoxic agent, such as cyclophosphamide or cyclosporine $\mathrm{A}$, has also been trialled with mixed success [21, 30-34]. Unfortunately, the lack of a control group for these "standard of care" treatments significantly restricts the conclusions that can be drawn, particularly about the impact of corticosteroids on the natural course of the disease.

In a retrospective analysis of 461 IPF patients, AEIPF without a known trigger occurred in 90 patients. Almost all patients received treatment with corticosteroids alone (65 out of $90,72 \%$ ) or in combination with a cytotoxic agent (23 out of $90,26 \%)$, with only two patients (2\%) receiving no treatment [11]. Overall mortality in this group was high, with $50 \%$ in-hospital mortality and median survival of only 2.2 months.

With the concerns around potential harm of immunosuppressing AEIPF patients, PAPIRIS et al. [25] incorporated a strategy of managing AEIPF using best supportive care without corticosteroids as well as ceasing any pre-existing immunosuppressive treatments. Of 85 IPF admissions, 24 met criteria for AEIPF without a known trigger. The in-hospital mortality of these patients treated without corticosteroids was again 50\%, with median survival 1.73 months. While not directly comparable, this rate of mortality was consistent with case reports incorporating corticosteroids, raising the question of whether corticosteroids actually impact on the natural history of the disease. Furthermore, a history of immunosuppression prior to AEIPF was again found to be a poor prognostic marker for survival.

\section{Conclusion}

In summary, AEIPF is a deadly complication of an already challenging disease. Unlike IPF, acute exacerbations may have a significant inflammatory component; however, the role of corticosteroids in AEIPF is far from certain. As maintenance treatment for IPF, they do not appear to prevent, and may actually increase, the risk of an AEIPF. In the acute treatment of an exacerbation, they are weakly recommended by international consensus; however, the lack of evidence makes it impossible to draw conclusions on their efficacy.

The choice to consider corticosteroids in AEIPF should be tailored to the individual patient based on the pattern and severity of disease, consideration of the underlying triggers, and a careful risk-to-benefit comparison. Other management for AEIPF includes a rigorous search for reversible triggers, excellent supportive care and, where possible, enrolment in a clinical trial. AEIPF prevention is centred on the impedance of FVC decline with antifibrotic therapy, prompt treatment of potential triggers such as gastro-oesophageal reflux and infection, and the avoidance of harmful therapies such as combination prednisone and azathioprine. Recent advances in the definition and pathophysiology of AEIPF are facilitating the development of large, randomised control trials, which will hopefully shed light on viable treatment options in this area of unmet clinical need.

\section{Affiliations}

\section{Christopher J. Brereton ${ }^{1,2}$, Helen E. Jo ${ }^{1,3}$}

${ }^{1}$ Dept of Respiratory Medicine, Royal Prince Alfred Hospital, Sydney, Australia. ${ }^{2}$ Clinical and Experimental Sciences, University of Southampton, Southampton, UK. ${ }^{3}$ Faculty of Medicine, University of Sydney, Sydney, Australia. 


\section{Conflict of interest}

C.J. Brereton has nothing to disclose. H.E. Jo has nothing to disclose.

\section{References}

1. Raghu G, Collard HR, Egan JJ, et al. An official ATS/ERS/JRS/ ALAT statement: idiopathic pulmonary fibrosis: evidence-based guidelines for diagnosis and management. Am J Respir Crit Care Med 2011; 183: 788-824

2. Richeldi L, Collard HR, Jones MG. Idiopathic pulmonary fibrosis. Lancet 2017; 389: 1941-1952.

3. King TE, Bradford WZ, Castro-Bernardini S, et al. A phase 3 trial of pirfenidone in patients with idiopathic pulmonary fibrosis. N EnglJ Med 2014; 370: 2083-2092.

4. Richeldi L, du Bois RM, Raghu G, et al. Efficacy and safety of nintedanib in idiopathic pulmonary fibrosis. N EnglJ Med 2014 370: 2071-2082.

5. Lancaster L, Crestani B, Hernandez P, et al. Safety and survival data in patients with idiopathic pulmonary fibrosis treated with nintedanib: pooled data from six clinical trials. BMJ Open Respir Res 2019; 6: e000397

6. Collard HR, Ryerson CJ, Corte TJ, et al. Acute exacerbation of idiopathic pulmonary fibrosis. An international working group report. Am J Respir Crit Care Med 2016; 194: 265-275

7. Collard HR, Moore BB, Flaherty KR, et al. Acute exacerbations of idiopathic pulmonary fibrosis. Am J Respir Crit Care Med 2007; 176: 636-643.

8. Kreuter M, Koegler H, Trampisch M, et al. Differing severities of acute exacerbations of idiopathic pulmonary fibrosis (IPF) insights from the INPULSIS® trials. Respir Res 2019; 20: 71

9. Churg A, Müller NL, Silva $\mathrm{Cl}$, et al. Acute exacerbation (acute lung injury of unknown cause) in UIP and other forms of fibrotic interstitial pneumonias. Am J Surg Pathol 2007; 31: 277-284

10. Atkins CP, Loke YK, Wilson AM. Outcomes in idiopathic pulmonary fibrosis: a meta-analysis from placebo controlled trials. Respir Med 2014; 108: 376-387.

11. Song JW, Hong SB, Lim CM, et al. Acute exacerbation of idiopathic pulmonary fibrosis: incidence, risk factors and outcome. Eur Respir J 2011; 37: 356-363.

12. Konishi K, Gibson KF, Lindell KO, et al. Gene expression profiles of acute exacerbations of idiopathic pulmonary fibrosis. Am J Respir Crit Care Med 2009; 180: 167-175.

13. Schupp JC, Binder $\mathrm{H}$, Jäger $B$, et al. Macrophage activation in acute exacerbation of idiopathic pulmonary fibrosis. PLoS One 2015; 10: e0116775.

14. Lee JS, Song JW, Wolters PJ, et al. Bronchoalveolar lavage pepsin in acute exacerbation of idiopathic pulmonary fibrosis. Eur Respir J 2012; 39: 352-358.

15. Wootton SC, Kim DS, Kondoh Y, et al. Viral infection in acute exacerbation of idiopathic pulmonary fibrosis. Am J Respir Crit Care Med 2011; 183: 1698-1702.

16. Johannson KA, Vittinghoff E, Lee K, et al. Acute exacerbation of idiopathic pulmonary fibrosis associated with air pollution exposure. Eur Respir J 2014; 43: 1124-1131.

17. Suzuki H, Sekine $Y$, Yoshida S, et al. Risk of acute exacerbation of interstitial pneumonia after pulmonary resection for lung cancer in patients with idiopathic pulmonary fibrosis based on preoperative high-resolution computed tomography. Surg Today 2011; 41: 914-921.

18. Sakamoto K, Taniguchi $\mathrm{H}$, Kondoh $\mathrm{Y}$, et al. Acute exacerbation of IPF following diagnostic bronchoalveolar lavage procedures. Respir Med 2012; 106: 436-442.
19. Raghu G, Anstrom KJ, King TE, et al. Prednisone, azathioprine, and $N$-acetylcysteine for pulmonary fibrosis. N EnglJ Med 2012; 366: 1968-1977.

20. Collard HR, Richeldi L, Kim DS, et al. Acute exacerbations in the INPULSIS trials of nintedanib in idiopathic pulmonary fibrosis. Eur Respir J 2017; 49: 1601339.

21. Akira M, Kozuka T, Yamamoto $\mathrm{S}$, et al. Computed tomography findings in acute exacerbation of idiopathic pulmonary fibrosis. Am J Respir Crit Care Med 2008; 178: 372-378.

22. Akira $M$, Hamada $H$, Sakatani $M$, et al. CT findings during phase of accelerated deterioration in patients with idiopathic pulmonary fibrosis. AJR Am J Roentgenol 1997; 168: 79-83.

23. Ranieri VM, Rubenfeld GD, Thompson BT, et al. Acute respiratory distress syndrome: the Berlin Definition. JAMA 2012; 307: 2526-2533.

24. Annane D, Pastores SM, Rochwerg B, et al. Guidelines for the diagnosis and management of critical illness-related corticosteroid insufficiency $(\mathrm{CIRCI})$ in critically ill patients (part I): Society of Critical Care Medicine (SCCM) and European Society of Intensive Care Medicine (ESICM) 2017. Crit Care Med 2017; 45: 2078-2088.

25. Papiris SA, Kagouridis K, Kolilekas L, et al. Survival in idiopathic pulmonary fibrosis acute exacerbations: the nonsteroid approach. BMC Pulm Med 2015; 15: 162.

26. Kondoh Y, Taniguchi $\mathrm{H}$, Kawabata $\mathrm{Y}$, et al. Acute exacerbation in idiopathic pulmonary fibrosis. Analysis of clinical and pathologic findings in three cases. Chest 1993 103: 1808-1812.

27. Al-Hameed FM, Sharma S. Outcome of patients admitted to the intensive care unit for acute exacerbation of idiopathic pulmonary fibrosis. Can Respir /2004; 11: 117-122.

28. Tachikawa R, Tomii $\mathrm{K}$, Ueda $\mathrm{H}$, et al. Clinical features and outcome of acute exacerbation of interstitial pneumonia: collagen vascular diseases-related versus idiopathic. Respiration 2012; 83: 20-27

29. Kondoh Y, Azuma A, Inoue $Y$, et al. Thrombomodulin alfa for acute exacerbation of idiopathic pulmonary fibrosis. A randomized, double-blind placebo-controlled trial. Am J Respir Crit Care Med 2020; 201: 1110-1119

30. Fujimoto $\mathrm{K}$, Taniguchi $\mathrm{H}$, Johkoh $\mathrm{T}$, et al. Acute exacerbation of idiopathic pulmonary fibrosis: high-resolution CT scores predict mortality. Eur Radiol 2012; 22: 83-92.

31. Donahoe M, Valentine VG, Chien N, et al. Autoantibodytargeted treatments for acute exacerbations of idiopathic pulmonary fibrosis. PLoS One 2015; 10: e0127771.

32. Yokoyama $\mathrm{T}$, Kondoh $\mathrm{Y}$, Taniguchi $\mathrm{H}$, et al. Noninvasive ventilation in acute exacerbation of idiopathic pulmonary fibrosis. Intern Med 2010; 49: 1509-1514.

33. Okamoto $\mathrm{T}$, Ichiyasu $\mathrm{H}$, Ichikado $\mathrm{K}$, et al. [Clinical analysis of the acute exacerbation in patients with idiopathic pulmonary fibrosis]. Nihon Kokyuki Gakkai Zasshi 2006; 44: 359-367.

34. Ambrosini V, Cancellieri A, Chilosi M, et al. Acute exacerbation of idiopathic pulmonary fibrosis: report of a series. Eur Respir J 2003; 22: 821-826. 